\title{
The continued fight for equity
}

\author{
On the occasion of Pride month, we take the opportunity to revisit the discussion about equity in science and \\ beyond and what we are doing to help.
}

A s millions of LGBTQI+ people around the globe are celebrating Pride month and the notable achievements of that movement, we reflect on the continued struggle to achieve diversity, equity and inclusion (DEI) in our everyday habits, our institutes, around our dinner tables, in our governments, on the boards of directors of our companies, and everywhere in between.

The first stop in any discussion about DEI is usually gender representation in the workplace. Nature Astronomy has published two special issues on the topic of women in astronomy and best practices for improving DEI in astronomy and science in general. In this issue, we publish a study by Lisa Kewley that forward-models the fraction of women in the astronomy workforce in Australia. The results are demotivating: current inequities in hiring practices along with significantly higher attrition rates for women mean that the pretty tame goal of $30 \%$ women at all levels of academia - a goal set out in the 2016-2025 Decadal Plan for Australian Astronomy - won't be achieved for another 60 years. Even if affirmative action were implemented consistently and throughout the community, another 10 years would be required to reach that target.

The attrition rate of women in academia is the consequence of a multitude of different processes that work against them. Pauline Leonard discusses some of the issues women face in their workplaces in a News \& Views that accompanies Kewley's paper. Microaggressions - instances of indirect, subtle or unintentional discrimination - are the way by which "sexism and discrimination are thoroughly embedded in the infrastructures of academic departments." No amount of high-level policy making can alleviate this (career) death by a thousand cuts.

As Leonard points out, "To change cultures of marginalization and oppression, leadership that fully acknowledges the overt and covert ways in which these are operationalized is essential." At the highest levels of international policy making, the United Nations Sustainable Development Goals (SDGs) have enshrined gender equality as one of the goals necessary for humanity's sustainable survival. How this top-down approach translates into action on the intermediate- and small-scale theatres of life is not always obvious. At Springer Nature, we have been publishing our sustainable business reports since 2018. In these reports, we track our progress towards some of these SDGs, including gender equality and impact on climate. Our latest report, published in early April 2021, shows that we are on track for achieving our goal of $45 \%$ women in positions of leadership by 2023 .

In addition to leading by example, academic publishers are in the unique position of being able to actively promote gender equity in the communities they serve, both by facilitating the dissemination of knowledge in an equitable way (see, for example, our commitment to open access) but also by collecting, curating and presenting relevant content in ways that make knowledge available and accessible to scientists, policy and decision makers and the public. At the beginning of March, we launched our SDG 5 Gender Equality hub, bringing together content from across the Springer Nature journals, books and magazines. The launching theme for the hub was 'Women in Science', though expect an update this month to highlight the intersection between gender and sexuality.

Along similar lines is also the Nature Research Awards for Inspiring Women in Science, with two categories focusing on Science Outreach and Science Achievement, respectively. The inaugural award for science outreach in 2018 was awarded to Mirjana Pović, an astronomer, for her work on the development of science and education in sub-Saharan Africa. The deadline for applications this year is fast approaching on 20 June and we are excited to see the next cohort of inspiring women in science (dare we hope, astronomers?), cognizant as we are of the importance of role models in establishing positive feedback loops to increase representation.

The discussion about DEI, however, should not stop at gender. Humans have been destructively imaginative in identifying arbitrary ways of discriminating against the 'other'. Racism is a constant presence in the lives of people of colour, and the abysmal representation of scientists of colour in astronomy and science overall is just one of the multiple facets of this issue. The struggle of scientists of colour to be seen and taken seriously is not new, as exemplified in the recently published memoirs of Katherine Johnson and Chanda Prescod-Weinstein two lived experiences several decades apart that are disappointingly similar. How can we do better? There are several different initiatives aimed at providing access to knowledge in underserved communities, both within developed countries and in the developing world, with some specifically targeting people of colour (see our recently published Comment by Linda Strubbe and collaborators on the Pan-African School for Emerging Astronomers and our living collection on Astronomy for Development).

However, bringing a more diverse group of people into astronomy is not enough. Keeping them there is the real challenge. In 2018, we introduced the Springer Nature Employee Networks, company-wide communities where people can connect and empower each other. Springer Nature Pride was our first such network that included LGBTQI+ employees and their allies. Over the last three years several more employee networks were founded, bringing together people of colour, people with disabilities and neuroatypical people, women, and parents. Each of these groups has since embarked on a journey of not only creating safe spaces for their members but also taking an active role in educating our company and informing our company's policies.

It is hard to imagine how different our scientific communities might have looked had we had such an understanding and appreciation of diversity since the birth of our scientific institutions. We would most certainly have more out and proud LGBTQI+ colleagues to celebrate Pride month with. Inequity and under-representation in the scientific community are only minor aspects of a much greater problem. Yet we have to start from somewhere. It is our universities that educate the future generations, and a diverse, equitable academic environment might just be one of the seeds of change that we can plant today for a different tomorrow.

Published online: 15 June 2021 https://doi.org/10.1038/s41550-021-01412-1 